\title{
Quiet-Sun variability observed with SUMER and CDS
}

\author{
A. Brković ${ }^{1,2}$, S. K. Solanki ${ }^{3}$, and I. Rüedi ${ }^{4}$ \\ 1 Institute of Astronomy, ETH-Zentrum, 8092 Zürich, Switzerland \\ 2 Kiepenheuer-Institut für Sonnenphysik, 79104 Freiburg, Germany \\ 3 Max-Planck-Institut für Aeronomie, Max-Planck-Str. 2, 37191 Katlenburg-Lindau, Germany \\ 4 Physikalisch-Meteorologisches Observatorium Davos, World Radiation Center, 7260 Davos Dorf, Switzerland \\ Received 16 October 2001 / Accepted 18 December 2001
}

\begin{abstract}
Brightness variations of solar features are investigated using time series of images and spectra of quietSun regions at disc centre obtained with the Coronal Diagnostic Spectrometer (CDS) and the Solar Ultraviolet Measurements of Emitted Radiation (SUMER) instrument onboard the SOHO spacecraft. Ultraviolet emission lines sampling temperatures of the chromosphere, transition region and corona were recorded, with the HeI $584.3 \AA$ and Ov $629.7 \AA$ lines being recorded simultaneously by both instruments. A comparison shows that both instruments give similar results except that SUMER reveals a factor of three higher absolute and relative variability than CDS. Simple tests suggest that the higher spatial resolution of SUMER compared to CDS, and the broad slit used for the CDS observations, are responsible for this difference. This points to the need for higher spatial resolution for future variability studies. The SUMER results confirm and extend to lower temperatures the trends deduced in an earlier paper from CDS data.
\end{abstract}

Key words. Sun: chromosphere - Sun: corona - Sun: transition region - Sun: UV radiation

\section{Introduction}

Heating processes in the quiet solar atmosphere are the consequences of magnetic reconnection or the dissipation of acoustic or magnetohydrodynamic waves which produce transient brightenings in the emission from the relevant atmospheric layers (e.g., Schadee et al. 1983; Lin et al. 1984; Parker 1988; Ulmschneider et al. 1991). These brightenings and their spatial and temporal variations are studied in the hope of improving our understanding of the heating mechanisms. A short overview of such, mainly observational, studies is given by Brković et al. (2000).

In this paper, we present the results of a statistical analysis of the brightness variations observed in the quiet Sun using movies obtained with CDS and time series of spectra recorded by SUMER. In particular, we compare the variability obtained by the two instruments in He I $584.3 \AA$ and $\mathrm{OV} 629.7 \AA$ at the same location (to the extent possible given the different resolutions of SUMER and CDS). Due to the uncertainty in the exact slit locations, such an analysis is probably best carried out with data of the type employed here, namely SUMER spectra and CDS wide-slit images. The present analysis is thus an extension of work presented by Brković et al. (2000), which was based exclusively on CDS data. Preliminary results of the present work were presented by Brković et al. (2001a).

Send offprint requests to: A. Brković, e-mail: alen@kis.uni-freiburg.de

\section{Observations}

The observations of quiet regions at Sun centre have been made using the Normal Incidence Spectrometer (NIS) of the Coronal Diagnostic Spectrometer (CDS) (Harrison et al. 1995) and the Solar Ultraviolet Measurements of Emitted Radiation (SUMER) spectrometer (Wilhelm et al. 1995) onboard the SOHO spacecraft. A list of the lines analysed, temperatures of line formation, signal-tonoise $(S / N)$ ratios, times of observations and exposure times are given in Tables 1 and 2. At the tabulated line formation temperatures the corresponding line emissivities are maximal under equilibrium conditions (Landini \& Monsignori Fossi 1990). The tabulated $S / N$ ratio is the average over all pixels and time steps. SUMER observations are designated with small letters, while the CDS ones are marked by capital letters. Earlier detailed comparisons between CDS and SUMER data have been carried out by Brynildsen et al. (1998) and Pauluhn et al. (1999, 2000).

\subsection{CDS observations}

NIS was employed in its movie mode, i.e., with a $90^{\prime \prime} \times$ $240^{\prime \prime}$ slit. In this mode a filtergram covering a part of the solar surface corresponding to the slit size is produced simultaneously at each wavelength. Due to the overlap of the images from neighbouring wavelengths, spectral information within each spectral line is lost. After correction for solar rotation, performed on the ground, each pixel 
Table 1. SUMER and CDS lines. Width refers to the width of the spectral window.

\begin{tabular}{|c|c|c|c|c|}
\hline SUMER lines $(\AA)$ & $\log \left(T_{\mathrm{e}} / \mathrm{K}\right)$ & Run No. & Width $(\AA)$ & $S / N$ ratio \\
\hline Не г 584.33 & 4.50 & $2 \mathrm{a}$ & 1.1 & 9.3 \\
\hline O v 629.73 & 5.39 & $2 b$ & 1.1 & 17.3 \\
\hline O vi 1031.91 & 5.50 & $1 \mathrm{a}, 3 \mathrm{a}$ & 2.2 & $10.0,10.3$ \\
\hline О г 1152.1 & 4.18 & $2 \mathrm{a}$ & 2.2 & 8.6 \\
\hline C I 1267.67 & 4.18 & $2 \mathrm{~b}$ & 2.1 & 7.3 \\
\hline O г 1302.17 & 4.18 & $1 b, 3 b$ & 1.1 & $9.2,10.6$ \\
\hline О г 1304.86 & 4.18 & $1 b, 3 b$ & 1.1 & $9.3,10.3$ \\
\hline Si II 1309.28 & 4.10 & $1 b, 3 b$ & 1.1 & $14.6,15.5$ \\
\hline N I 1319.00 & 4.21 & $1 b, 3 b$ & 1.1 & $8.6,8.8$ \\
\hline C II 1334.52 & 4.60 & $1 b, 3 b$ & 1.1 & $8.2,9.7$ \\
\hline \multicolumn{5}{|l|}{ CDS lines $(\AA)$} \\
\hline Mg IX 368.07 & 6.00 & $1 \mathrm{~A}, 2 \mathrm{~A}, 2 \mathrm{~B}, 3 \mathrm{~A}$ & 3.4 & $4.1,3.9,4.1,3.7$ \\
\hline Не г 584.33 & 4.50 & $1 \mathrm{~A}, 2 \mathrm{~A}, 2 \mathrm{~B}, 3 \mathrm{~A}$ & 5.8 & $11.7,10.4,10.2,10.4$ \\
\hline O v 629.73 & 5.39 & $1 \mathrm{~A}, 2 \mathrm{~A}, 2 \mathrm{~B}, 3 \mathrm{~A}$ & 5.8 & $8.0,7.0,7.1,7.2$ \\
\hline
\end{tabular}

Table 2. CDS (1A, 2A, 2B, 3A) and SUMER (1a, 1b, 2a, 2b, $3 \mathrm{a}, 3 \mathrm{~b})$ runs.

\begin{tabular}{|c|c|c|c|c|c|}
\hline $\begin{array}{l}\text { Run } \\
\text { No. }\end{array}$ & $\begin{array}{l}\text { Date } \\
1997 \\
\text { April }\end{array}$ & $\begin{array}{l}\text { Start } \\
\text { UT } \\
\text { time }\end{array}$ & $\begin{array}{l}\text { End } \\
\text { UT } \\
\text { time }\end{array}$ & $\begin{array}{c}\text { Exposure time (s) } \\
+ \\
\text { Overhead (s) }\end{array}$ & $\begin{array}{l}\text { Slit } \\
\text { No. }\end{array}$ \\
\hline $1 \mathrm{~A}$ & 22 & $07: 45$ & $18: 22$ & $15+5$ & 6 \\
\hline $1 \mathrm{a}$ & 22 & $09: 35$ & $13: 47$ & $15+4$ & 6 \\
\hline $1 b$ & 22 & $13: 52$ & 19:08 & $15+4$ & 3 \\
\hline $2 \mathrm{~A}$ & 23 & 08:01 & 11:49 & $10+4$ & 6 \\
\hline $2 \mathrm{a}$ & 23 & 08:04 & $13: 40$ & $15+4$ & 3 \\
\hline $2 \mathrm{~B}$ & 23 & $12: 58$ & $18: 55$ & $10+4$ & 6 \\
\hline $2 \mathrm{~b}$ & 23 & $13: 44$ & 18:43 & $30+4$ & 3 \\
\hline $3 \mathrm{~A}$ & 25 & 08:01 & $18: 55$ & $10+4$ & 6 \\
\hline $3 a$ & 25 & $12: 46$ & $18: 19$ & $15+4$ & 6 \\
\hline $3 b$ & 25 & 07:05 & 12:41 & $15+4$ & 3 \\
\hline
\end{tabular}

follows the same point on the solar surface during the whole time series. Thus, the CDS data analysed here are similar to those studied by Brković et al. (2000, 2001b), the main difference being that the present data have been taken at a faster rate (shorter exposure time). To the actual exposure time an overhead of four or five seconds per frame must be added (the overhead is mainly accrued by reading out the CCD and preparing it for the next exposure). Due to telemetry constraints, somewhat less than half of the data along the slit were read out and consequently only a smaller area of the solar disc is covered. The correction for solar rotation further reduces the size of the field of view, which finally is $70^{\prime \prime} \times 109^{\prime \prime}(42 \times 65)$ pixels, with a pixel size of $1.68^{\prime \prime} \times 1.68^{\prime \prime}$.

Note that during runs $1 \mathrm{~A}, 1 \mathrm{a}, 1 \mathrm{~b}$ roughly the same quiet solar region was recorded. The same is true for runs $2 \mathrm{~A}, 2 \mathrm{~B}, 2 \mathrm{a}, 2 \mathrm{~b}$ and for $3 \mathrm{~A}, 3 \mathrm{a}, 3 \mathrm{~b}$.

Due to the broad point-spread function of CDS, individual pixels are not independent in their brightness and noise properties (Thompson 1998). To account for this property we bin the intensities from groups of $4 \times 4$ pixels for the analysis described here. In this manner the $S / N$ ratio is increased by a factor of four, but the spatial sampling is proportionately reduced. Following Thompson (1998) the noise values for single pixel and groups of pixels have been multiplied by square root of two. The reason for this factor is that photon-related statistical noise in the NIS data has two sources of similar magnitude. One is the Poisson noise associated with the photons which interact with the detector. The second source is the fluctuation in the amount of amplification in the detector system. The signal-to-noise ratio for each single pixel, i.e., prior to the binning, and each time step is calculated as: $S / N=$

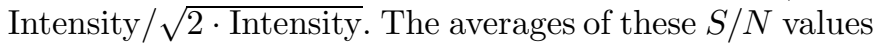
over all pixels and time steps result in $S / N$ ratios given in Table 1.

\subsection{SUMER observations}

SUMER observed simultaneously with CDS using $1^{\prime \prime} \times$ $120^{\prime \prime}$ slits \# 3 and \# 6 , with detector B and a pixel size of $1^{\prime \prime} \times 1^{\prime \prime}$. The SUMER slit was located within the CDS field of view and was kept at a fixed location on the solar surface by compensating for solar rotation. Due to the asymmetric slit and internal misalignments only $90^{\prime \prime}$ along the slit were of use, resulting in a final field of view of $1^{\prime \prime} \times 90^{\prime \prime}$. The observations cover chromospheric and transition region temperatures.

Several instrumental corrections have been applied to the data before the analysis. For the flat-field correction we used the flat-field image taken on 24 April 1997. The pin cushion distortion of the image and the inclination of the spectral lines with respect to the detector columns were removed. The effects of the dead time and gain depression of the detector were almost negligible, but the corrections have been applied anyway. In the next step we fit the line profiles at each spatial position and for each time step. For all lines least-squares fits of a single Gaussian plus a linear 

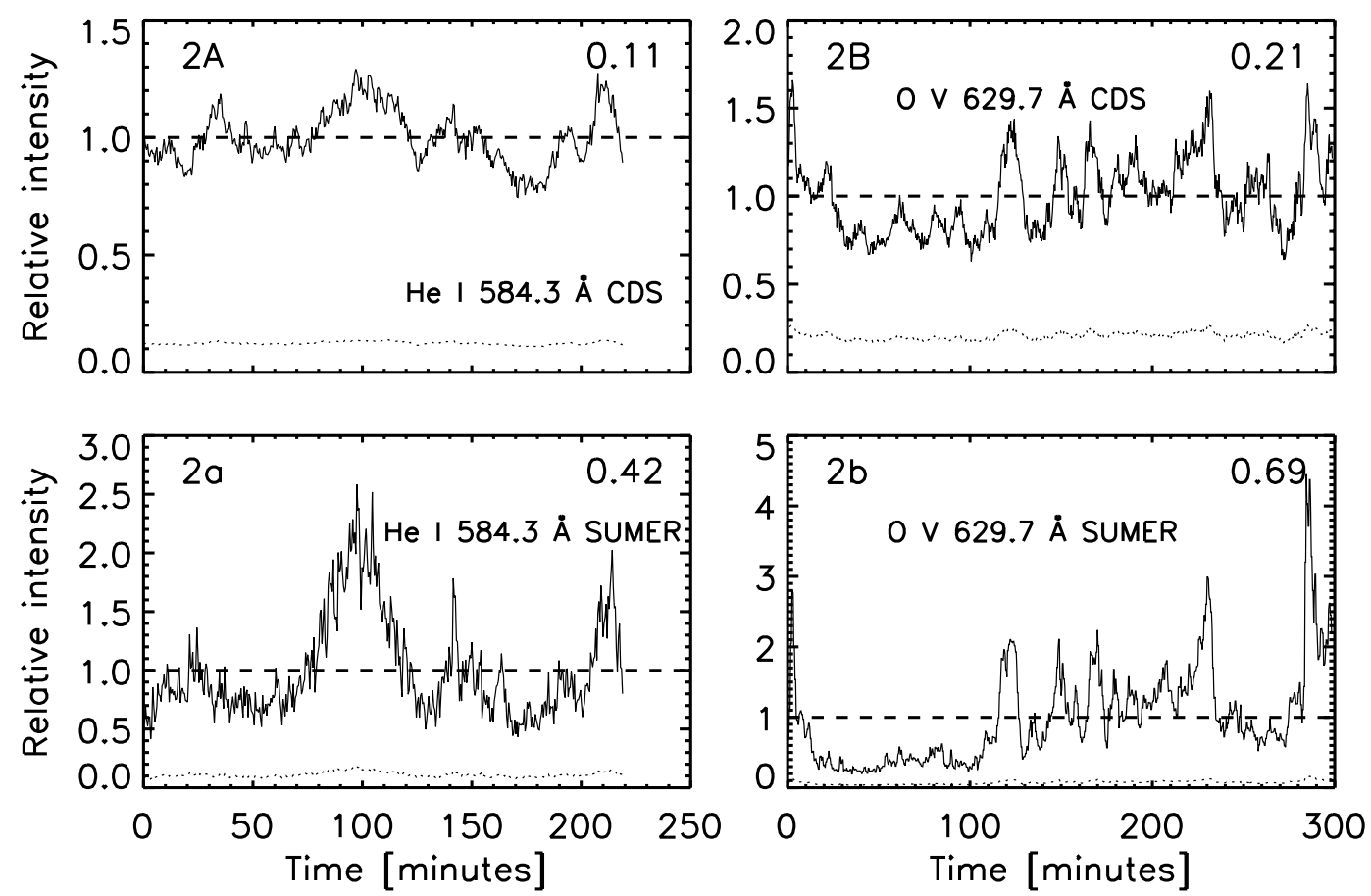

Fig. 1. Sample light curves for the He I and Ov lines of CDS (upper frames) and SUMER (lower frames). Plotted is the intensity relative to the normalized time-averaged intensity (the horizontal dashed lines) for individual pixels. Note the difference in scale between the SUMER and CDS light curves. The dotted lines represent noise levels. The run number and the relative variability are given at the upper left and right corners of each frame.

background turned out to be sufficient. After subtraction of the linear background the total line intensity, $I_{\text {tot }}$, is given by $\sqrt{\pi} I_{\mathrm{m}} \sigma$, where $\sigma$ is the Doppler width and $I_{\mathrm{m}}$ is the maximum of the spectral intensity of the fit. The temporal variations of this parameter are analysed here.

\subsection{Coalignment of SUMER and CDS}

Since we are interested in comparing co-spatial and cotemporal time series of $\mathrm{He}$ I and $\mathrm{O} v$ obtained with two instruments, the position of the SUMER slit in the CDS field of view had to be determined. In principle it would be sufficient to cross-correlate nearly simultaneous SUMER and CDS exposures to obtain the position of SUMER's slit in CDS's field of view. However such individual spatial crosscorrelations gave unreliable results, since the CDS image showed insufficient spatial structure. Hence, the position of a given pixel on the SUMER slit relative to the CDS image $\left(x_{\mathrm{S}}, y_{\mathrm{S}}\right)$ turned out to exhibit considerable scatter with time. Here $x_{\mathrm{S}}$ is the $\mathrm{E}-\mathrm{W}$ and $y_{\mathrm{S}}$ the $\mathrm{N}-\mathrm{S}$ location.

We therefore carried out a more elaborate procedure. For each $\left(x_{\mathrm{S}}, y_{\mathrm{S}}\right)$-pair the SUMER light curve of each pixel along the slit was cross-correlated with the corresponding CDS light curve. Since the intensity exhibits strong temporal variations this turned out to be a far more robust way of determining the coalignment. Then the average of the correlation coefficients obtained along the SUMER slit, $c_{\mathrm{S}}$, was determined. The $\left(x_{\mathrm{S}}, y_{\mathrm{S}}\right)$-pair for which $c_{\mathrm{S}}\left(x_{\mathrm{S}}, y_{\mathrm{S}}\right)$ is largest is the required location. The largest $c_{\mathrm{S}}$ values obtained in this manner are over 0.9 . Note that no background was subtracted either for CDS or for SUMER data. We used the SUMER data prior to the fitting procedure and for each pixel a spectral summation over the line profile was performed. The CDS data used in this step were the one prior to the $4 \times 4$ binning. Since the instruments do not have the same resolution along the slit the SUMER data were rebinned to the pixel size of CDS, i.e., $1.68^{\prime \prime}$.

The final $\left(x_{\mathrm{S}}, y_{\mathrm{S}}\right)$ pairs obtained are close to the time average of the $\left(x_{\mathrm{S}}(t), y_{\mathrm{S}}(t)\right)$ array obtained from simple spatial cross-correlations, which speaks for the robustness of the coalignment.

\section{Results}

First we give the definition of some parameters which are analysed in this work. The instantaneous intensity and noise were defined in Sects. 2.1 and 2.2. The variability is described by the rms of the time series. The (average) intensity and noise are averages over the whole duration of the observations. The relative variability is defined as the ratio of the rms to the intensity. These four parameters were determined for each pixel (SUMER) and for each group of $4 \times 4$ pixels (CDS) in a spectral line of interest. Sometimes we give a value of the relative intensity which is defined as the momentary intensity relative to the time-averaged intensity. Since the results obtained from the same spectral line observed on different days, in general, differ only insignificantly, the parameters 

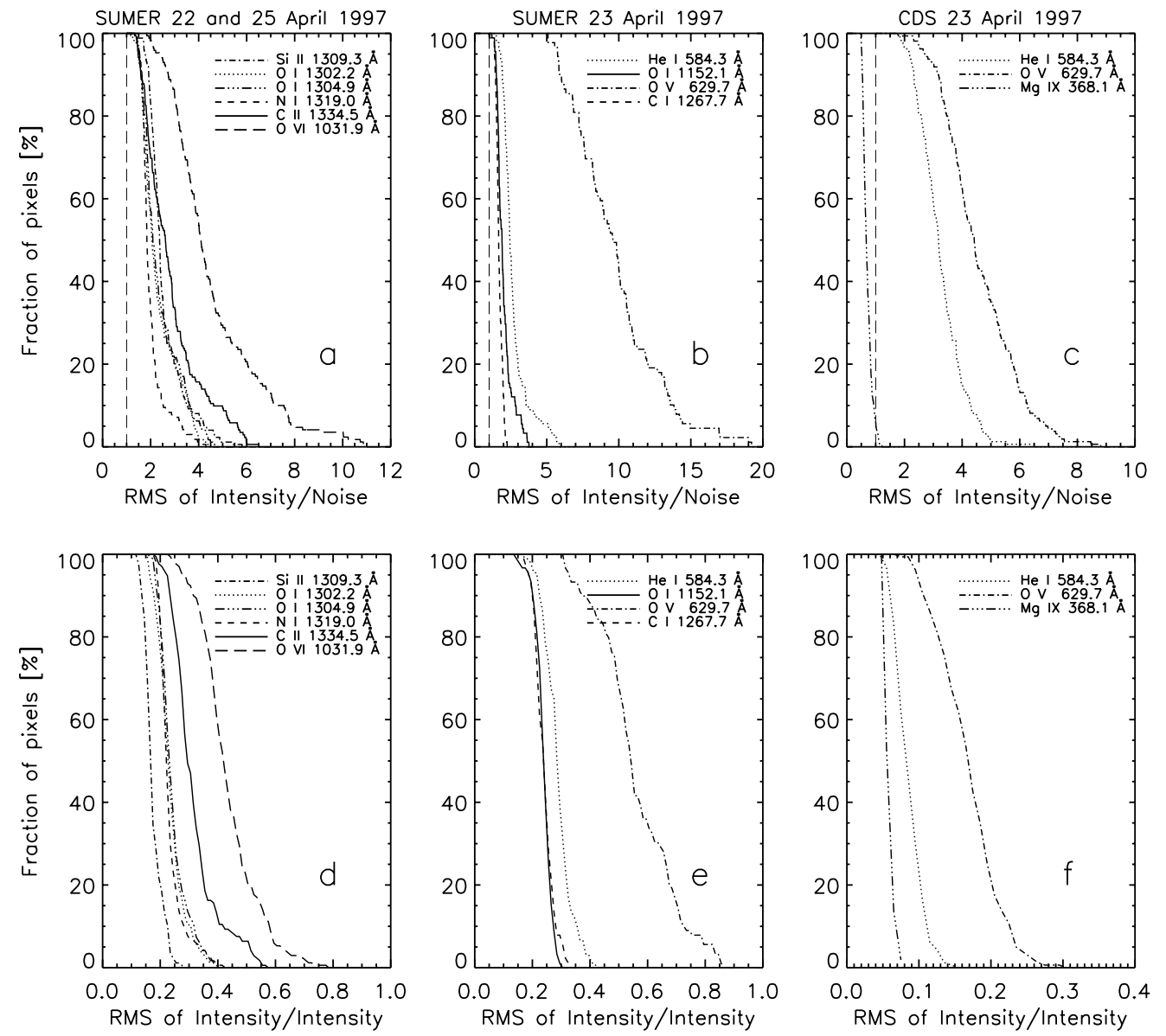

Fig. 2. Frames $\mathbf{a}-\mathbf{c}$ ) upper row: fraction of points with an intensity-rms to noise ratio lying above the corresponding value on the abscissa. The thin vertical dashed line marks rms values corresponding to the $1 \sigma$ noise level. Frames $\mathbf{d}-\mathbf{f})$ lower row: fraction of points with relative variability lying above the corresponding value on the abscissa.

obtained from these data were combined and then analysed as a single data set for the sake of improved statistics.

\subsection{Comparison of light curves}

Figure 1 shows examples of normalized light curves cospatially and co-temporally observed with SUMER and CDS. Note, however, that the plotted He I and Ov data are independent of each other since they were recorded at different times. Each light curve refers to a single pixel, so that the data have neither been grouped nor rebinned. The dashed lines correspond to time-averaged intensity while the dotted lines represent count noise levels, where the noise is determined in each time step as the square root of the intensity (SUMER) and the square root of twice the intensity (CDS), with the intensity now being the instantaneous intensity. The relative variability is given in the upper right corner of each frame. Clearly, the SUMER data are much more variable than the CDS data. This is true for both spectral lines. SUMER exhibits an excess variability at all locations along the slit. The high $S / N$ ratio of the SUMER data and the good correlations between the SUMER and CDS data sets rule out noise as the source of this excess variability.

\subsection{Comparison of brightness variations between $C D S$ and SUMER}

To test the significance of the variability, we plot in Figs. $2 \mathrm{a}-\mathrm{c}$ the percentage of pixels with an rms/noise ratio lying above the corresponding value on the abscissa at every point within the field of view. The results obtained with SUMER (two left frames) are plotted separately from those obtained with CDS (rightmost frame). The rms/noise ratio is above 1.0 for chromospheric lines and above 2.0 and 5.0 for the transition region $\mathrm{O}$ VI and Ov lines for all pixels (similar results are obtained in Rabin \& Dowdy 1992; Berghmans et al. 1998; Brković et al. 2000). Thus, all intensity variations are larger than the noise and consequently real. The only exception to this rule is the $\mathrm{Mg}$ IX line in which less than $6 \%$ of the points are significantly variable at the $1 \sigma$ level, compared to $47 \%$ in datasets recorded in December 1996 (Brković et al. 2000). This difference is due to the higher noise introduced by the shorter exposure time of the new data. Hence it should be kept in mind that the results obtained from this line are less significant. Note that due to the noise contribution the measured variability is actually an upper limit to the true variability in this line. The remaining 

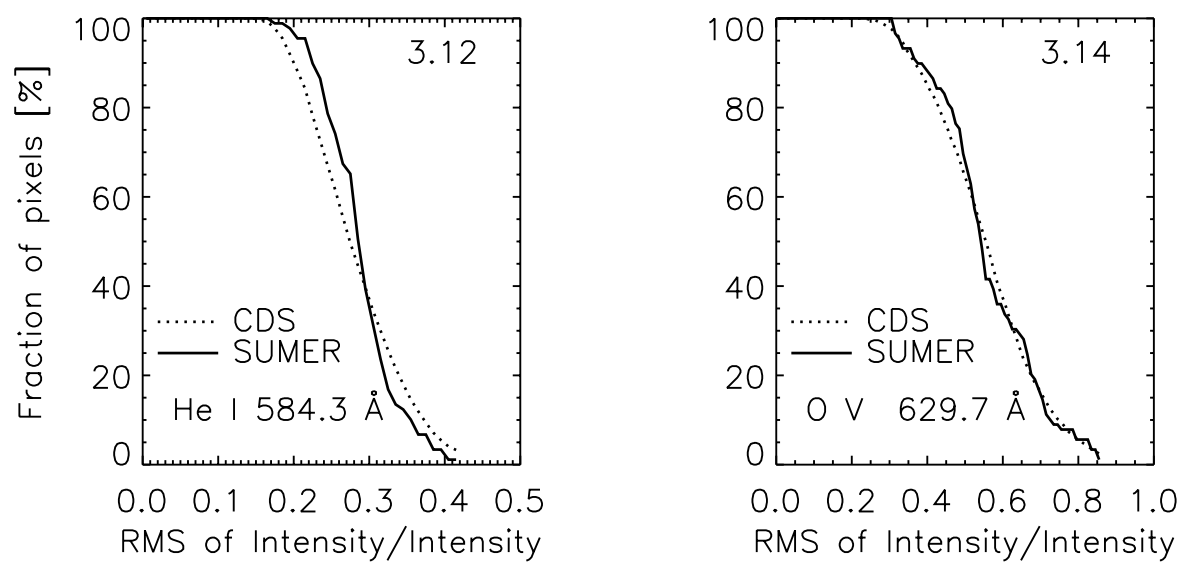

Fig. 3. Fits between CDS and SUMER relative variabilities. Proportionality factors are given at the upper right corner of each frame.

CDS results are in agreement with those in Brković et al. (2000).

Figures $2 \mathrm{~d}-\mathrm{f}$ are similar to Figs. $2 \mathrm{a}-\mathrm{c}$, but display the relative variability (i.e., rms/intensity). The greater number of lines available in the SUMER observations clearly confirms that the relative variability is greatest in the transition region and smallest in coronal lines.

We now turn to the question of how much larger the variability exhibited by SUMER data is compared to CDS. A comparison of median variabilities, $9.5 \%$ (23.5\%) for $\mathrm{He}_{\mathrm{I}}$ and $17.5 \%$ (55.0\%) for $\mathrm{OV}$ as seen with CDS (SUMER) gives ratios of 2.5 and 3.1 between the two instruments for the two lines, respectively. On the other hand, the average relative variabilities are $9 \%$ (28\%) for He I and $16 \%$ (56\%) for $\mathrm{O} v$, for CDS (SUMER). This gives ratios of 3.1 and 3.5 between the two instruments.

A possibly better way of determining this factor is to stretch the abscissa of Fig. 2f until the $\chi^{2}$ between the $\mathrm{He}$ I and $\mathrm{Ov}$ relative variability obtained by the two instruments is minimised. The best fit curves (CDS results obtained prior to the $4 \times 4$ binning) are plotted in Fig. 3 . Proportionality factors are given at the upper right corner of each frame. This analysis implies that the variability exhibited by SUMER data is approximately a factor of 3.1 larger than that seen in CDS.

We checked by how much SUMER variabilities change if broad stretches of continuum which include the total widths of the spectral windows, are not removed from the SUMER data, i.e., if we sum spectrally over the line profile and the surrounding continuum instead of applying a Gaussian fit. As expected, the variability is reduced, although not equally for the two lines: the new average variabilities for $\mathrm{He}$ I and $\mathrm{O} v$ amount to 0.21 and 0.48 , respectively, which corresponds to reductions of $33 \%(0.28 / 0.21)$ and $17 \%(0.56 / 0.48)$. The proportionality factors between SUMER and CDS variability are then also reduced, becoming 2.38 and 2.66 for He I and $\mathrm{O}$ v, respectively. Thus the larger variability detected in SUMER data compared to CDS is partly due to the width of the slits used and the fact that we cannot separate between line and continuum variability in the CDS data. The widths of spectral
Table 3. Correlation coefficients between variabilities of CDS line pairs.

\begin{tabular}{lcccccc}
\hline Run No. & $X$ & $Y$ & $1 \mathrm{~A}$ & $2 \mathrm{~A}$ & $2 \mathrm{~B}$ & $3 \mathrm{~A}$ \\
\hline He $\mathrm{I}-\mathrm{O} \mathrm{V}$ & 0.77 & 0.86 & 0.78 & 0.70 & 0.60 & 0.61 \\
$\mathrm{He}_{\mathrm{I}}-\mathrm{Mg}_{\mathrm{IX}}$ & 0.53 & 0.65 & 0.64 & 0.15 & 0.27 & 0.70 \\
$\mathrm{O}_{\mathrm{V}-\mathrm{Mg} \mathrm{IX}}$ & 0.45 & 0.64 & 0.40 & 0.11 & 0.18 & 0.37 \\
\hline
\end{tabular}

windows for $\mathrm{He}$ I and $\mathrm{O} v$ lines amount to $1.1 \AA$ (SUMER) and $5.8 \AA$ (CDS).

Nevertheless, the major part of the difference between the two instruments is probably due to the difference in spatial resolution element. To test this, we spatially average the SUMER data over an increasing number of pixels and determine the variability of the averaged light curves until we obtain the CDS variability. The appropriate number turned out to be 35 pixels along the slit for both lines. This corresponds to roughly the same solar surface area as covered by $4 \times 4 \mathrm{CDS}$ pixels, although the geometry is different. Brković et al. (2000) showed that CDS relative variabilities are marginally affected by the $4 \times 4$ binning, which, as explained in Sect. 2.1, is due to the broad point-spread function. In what follows we revert to using SUMER data with the background removed.

\subsection{Do intensity variations in different lines occur simultaneously?}

Tables 3 and 4 give correlation coefficients between variabilities of various simultaneously recorded line pairs. Runs $X$ and $Y$ refer to additional CDS observations carried out on 3 and 6 December 1996, (see Brković et al. 2000).

The largest correlations in CDS data sets are for the He $\mathrm{I}-\mathrm{O} v$ pair. The variabilities of the two lines are well correlated in all datasets. For the other two pairs, i.e., $\mathrm{He} \mathrm{I}-\mathrm{Mg} \mathrm{IX}$ and $\mathrm{O} \mathrm{V}-\mathrm{Mg} \mathrm{IX}$, the correlation coefficients can differ strongly from one run to another. The explanation for the small correlation coefficients for the dataset of 

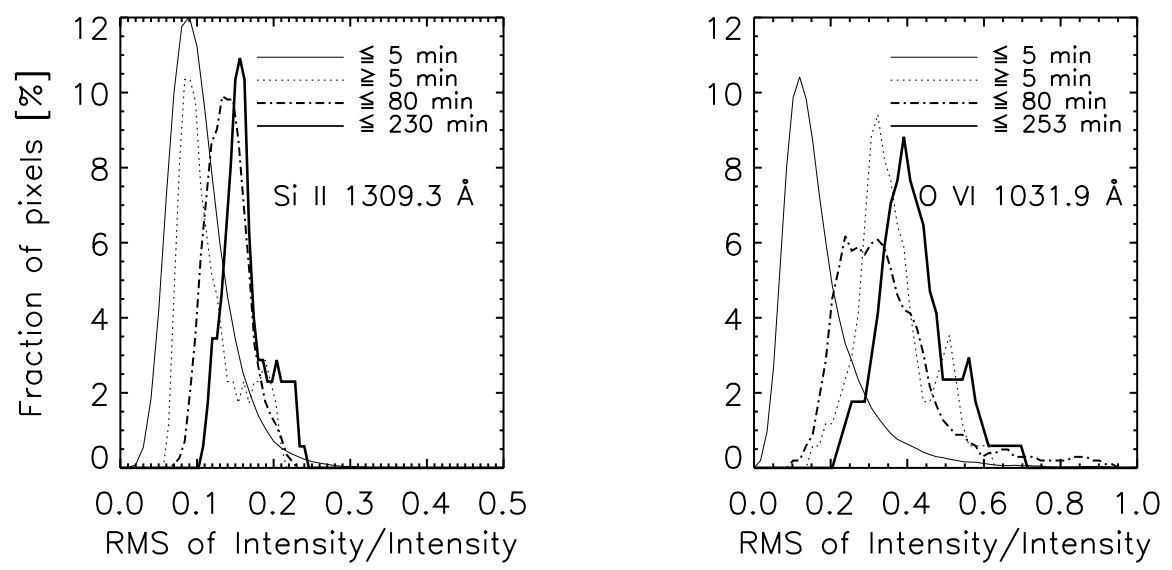

Fig. 4. Histograms of relative intensity fluctuations for two SUMER lines.

Table 4. Correlation coefficients between variabilities of SUMER line pairs.

\begin{tabular}{|c|c|}
\hline Line pair & Correlation coefficient \\
\hline Не г $584.33-\mathrm{O}_{\text {I }} 1152.10$ & $0.85^{2 \mathrm{a}}$ \\
\hline O v $629.73-\mathrm{C}_{\text {I }} 1267.67$ & $0.80^{2 \mathrm{~b}}$ \\
\hline О г $1302.17-$ о г 1304.86 & $0.97^{1 \mathrm{~b}}, 0.67^{3 \mathrm{~b}}$ \\
\hline O г $1302.17-$ Si i 1309.28 & $0.94^{1 \mathrm{~b}}, 0.89^{3 \mathrm{~b}}$ \\
\hline O I $1302.17-\mathrm{N}_{\text {I }} 1319.00$ & $0.27^{1 \mathrm{~b}}, 0.67^{3 \mathrm{~b}}$ \\
\hline О г $1302.17-\mathrm{C}$ п 1334.52 & $0.95^{1 \mathrm{~b}}, 0.80^{3 \mathrm{~b}}$ \\
\hline O г $1304.86-$ Si I 1309.28 & $0.91^{1 \mathrm{~b}}, 0.72^{3 \mathrm{~b}}$ \\
\hline O г $1304.86-\mathrm{N}_{\text {i }} 1319.00$ & $0.43^{1 \mathrm{~b}}, 0.45^{3 \mathrm{~b}}$ \\
\hline О г $1304.86-\mathrm{C}$ пг 1334.52 & $0.93^{1 \mathrm{~b}}, 0.67^{3 \mathrm{~b}}$ \\
\hline Si in $1309.28-\mathrm{N}_{\text {I }} 1319.00$ & $0.24^{1 \mathrm{~b}}, 0.68^{3 \mathrm{~b}}$ \\
\hline Si II $1309.28-$ C II 1334.52 & $0.88^{1 \mathrm{~b}}, 0.81^{3 \mathrm{~b}}$ \\
\hline N i $1319.00-\mathrm{C}$ II 1334.52 & $0.26^{1 \mathrm{~b}}, \quad 0.38^{3 \mathrm{~b}}$ \\
\hline
\end{tabular}

23 April 1997 (2A and 2B) could be that the barely significant variability seen in the $\mathrm{Mg}$ Ix line is even less reliable in these than in the other observations due to the decreased exposure time and consequently increased count noise (cf. Figs. 2a-c). As already noted by Brković et al. (2000), the $\mathrm{Mg}$ IX variability correlates better with $\mathrm{He}$ I than with $\mathrm{O} v$, probably due to the fact that the He I line is also sensitive to coronal properties (Fontenla et al. 1993; Andretta \& Jones 1997), and consequently a part of the fluctuations seen in He I is actually coronal in origin.

Correlation coefficients between variabilities of SUMER lines are all very high, with the exception of line pairs involving N I $1319.0 \AA$ (Table 4). The profiles of this line do not reveal anything unusual that could cause this lower correlation (e.g., signs of blending). Its singular behaviour remains not understood, since even a hidden blend should not lead to such a drastic reduction in correlation with the other lines, unless possibly it is coronal in origin. All lines, except the $\mathrm{O} v$ line are formed at chromospheric temperatures and the high correlation between their variabilities is not surprising. The very good correlation between $\mathrm{C}_{\mathrm{I}}$ and $\mathrm{OV}$ (even higher than that between $\mathrm{He}$ I and $\mathrm{Ov}$, Table 3 ) confirms the close connection between chromospheric and transition region variability.

In their study of quiet-Sun brightness variations, Brković et al. (2000) have found that the relative intensity variations of the $\mathrm{He}$ I, O v and $\mathrm{Mg}$ Ix lines do not depend significantly on the absolute intensity (i.e., the variability scales linearly with intensity). The present analysis confirms this result for both SUMER and CDS data. Thus the relative brightness fluctuations are equally strong in the network and in the intranetwork. The same result was found by Vernazza et al. (1975) for the chromospheric and transition region emission lines they investigated.

\subsection{Time scales of intensity variations}

Next we investigate, in a very simple manner, the time scales of the variability. In order to determine the relative brightness fluctuations occuring over time scales of $N$ min or less, we cut our observing time sequences into $N$ min pieces. For each of these we determine the rms of the intensity at each pixel (SUMER) and at each group of $4 \times 4$ pixels (CDS). For the relative brightness fluctuations occuring on time scales longer than $M$ min we determined the average intensity observed in each $M$ min interval and determined the rms of these values. The durations of the different time series lie in the range $230 \mathrm{~min}$ to $660 \mathrm{~min}$. We tested the influence of this duration by analysing only the first $230 \mathrm{~min}$ of the longer time series, but found the results to be unchanged. This is because the brightness fluctuations occur mainly on time scale below $80 \mathrm{~min}$ (see below).

Figure 4 shows histograms of the relative intensity fluctuations exhibited by two SUMER lines on different time scales. It displays the percentage of pixels with a relative intensity variability corresponding to the value on the abscissa. For the chromospheric Si II line fluctuations on time scales $\leq 5 \mathrm{~min}$ and those on time scales $\geq 5 \mathrm{~min}$ are almost equally strong. All chromospheric lines except He I $584.3 \AA$, which is optically thick and susceptible to coronal radiation, exhibit such a behaviour to some degree. He I behaves similarly to lines formed at higher temperatures, 
represented by $\mathrm{O} v \mathrm{i}$ in Fig. 4. For these the relative intensity fluctuations at time scales $\geq 5$ min dominate over those at shorter time scales. By considering a finer grid of time scales we find that the brightness fluctuations in the transition region and corona occur dominantly on time scale between 5 and 80 min. The transition region lines exhibit the largest variability on all investigated time scales while the coronal line (not shown) is least variable (in agreement with Vernazza et al. 1975; Brković et al. 2000).

We find that the ratio between the variability of the He I line seen by SUMER to that deduced from CDS data (see Sect. 3.2) is larger for shorter time scales than for the longer ones, while this factor does not change significantly for the $\mathrm{Ov}$ line. This suggests that the He I variations on shorter time scales are localized on smaller spatial scales than in $\mathrm{O} v$.

\section{Conclusions}

We have investigated the brightness variations in the quiet Sun using temporally and spatially overlapping time series obtained by CDS and SUMER in chromospheric, transition region and coronal lines.

The relative intensity variations are larger for the transition region lines than those of the chromospheric lines. The variations of the coronal Mg Ix line are even smaller, but recall that this line has the smallest $S / N$ ratio of all observed lines. The strength of the detected relative intensity variations is over a factor of three larger in SUMER data than in corresponding CDS recordings. This can be explained by the smaller resolution elements of SUMER compared to CDS and to a smaller extent by the wider slit used when obtaining the CDS data (due to which the continuum variability could not be separated from the line variability). The larger variability in SUMER than in CDS data is in agreement with the findings of Pauluhn et al. (2000).

In the upper chromosphere all parts of the quiet Sun, from the darkest intranetwork to the brightest network, are significantly variable at least at the $1 \sigma$ level, in agreement with the findings of Rabin \& Dowdy (1992), Berghmans et al. (1998) and Brković et al. (2000). In transition region lines of the O VI and Ov spectra the significance level of the variability lies above $2 \sigma$ and $5 \sigma$, respectively. This confirms that all fluctuations measured in the chromosphere and transition region are real intensity variations. In the corona, as sampled by Mg IX $368.1 \AA$, less than half of the points show significant variability at the $1 \sigma$ level.

It is confirmed that the relative variability is independent of the brightness at all studied temperatures. The brightness fluctuations observed in all non-chromospheric lines are dominantly due to brightness changes on time scales less than $80 \mathrm{~min}$ and longer than $5 \mathrm{~min}$. In all observed chromospheric lines, except the He I line, the brightness changes on time scales longer than $5 \mathrm{~min}$ are equally strong compared to the changes on time scales less than $5 \mathrm{~min}$.
Variabilities of different lines are highly correlated, except for the N I and Mg IX lines. These good correlations are in favour of a strong connection between brightenings in the chromosphere and transition region. The low correlation with $\mathrm{N}_{\mathrm{I}}$ is not understood, while the low correlation with $\mathrm{Mg}$ IX may be due to the lower significance of the variability of that line. This cannot be the entire cause for the lower correlation, however, since Brković et al. (2001b) also found a low correlation between blinkers (i.e. strong brightenings) seen in $\mathrm{O} v$ and $\mathrm{Mg}$ IX, although these brightenings were significant at the $3 \sigma$ level in both lines.

Acknowledgements. We thank J. O. Stenflo and M. C. E. Huber for their encouragement and support. We are also grateful to the SUMER and CDS teams as well as the SOHO command staff. Their help was invaluable in obtaining these observations. AB specially thanks K. Stucki, U. Schühle and A. Pauluhn for helping with the SUMER data acquisition and handling. SOHO is a mission of international cooperation between ESA and NASA. We thank K. Wilhelm for carefully reading and thoroughly commenting on the manuscript. This work was partly supported by the Swiss National Science Foundation, grant No. 21-45083.95, and by a grant from the ETH-Zürich, which is gratefully acknowledged.

\section{References}

Andretta, V., \& Jones, H. P. 1997, ApJ, 489, 375

Berghmans, D., Clette, F., \& Moses, D. 1998, A\&A, 336, 1039

Brković, A., Rüedi, I., Solanki, S. K., et al. 2000, A\&A, 353, 1083

Brković, A., Solanki, S. K., \& Rüedi, I. 2001a, in Recent Insights into the Physics of the Sun and HeliosphereHighlights from SOHO and Other Space Missions, ed. P. Brekke, B. Fleck, \& J. B. Gurman, ASP Conf. Ser., 200, 318

Brković, A., Solanki, S. K., \& Rüedi, I. 2001b, A\&A, 373, 1056 Brynildsen, N., Brekke, P., Fredvik, T., et al. 1998, Solar Phys., 179,279

Fontenla, J. M., Avrett, E. H., \& Loeser, R. 1993, ApJ, 406, 319

Harrison, R. A., Sawyer, E. C., Carter, M. K., et al. 1995, Solar Phys., 162, 233

Landini, M., \& Monsignori Fossi, B. C. 1990, A\&AS, 82, 229

Lin, R. P., Schwartz, R. A., Kane, S. R., Pelling, R. M., \& Hurley, K. C. 1984, ApJ, 283, 421

Parker, E. N. 1988, ApJ, 330, 474

Pauluhn, A., Rüedi, I., Solanki, S. K., et al. 1999, Appl. Opt., 38,7035

Pauluhn, A., Solanki, S. K., Rüedi, I., Landi, E., \& Schühle, U. 2000, A\&A, 362, 737

Rabin, D., \& Dowdy, J. F. Jr. 1992, ApJ, 398, 665

Schadee, A., De Jager, C., \& Śvestka, Z. 1983, Solar Phys., 89, 287

Thompson, W. 1998, Deriving Statistics from NIS Data, CDS Software Note No. 49

Ulmschneider, P., Priest, E. R., \& Rosner, R. (eds.) 1991, Mechanisms of Chromospheric and Coronal Heating (Springer-Verlag, Berlin)

Vernazza, J. E., Foukal, P. V., Huber, M. C. E., et al. 1975, ApJ, 199, L123

Wilhelm, K., Curdt, W., Marsch, E., et al. 1995, Solar Phys., 162,189 